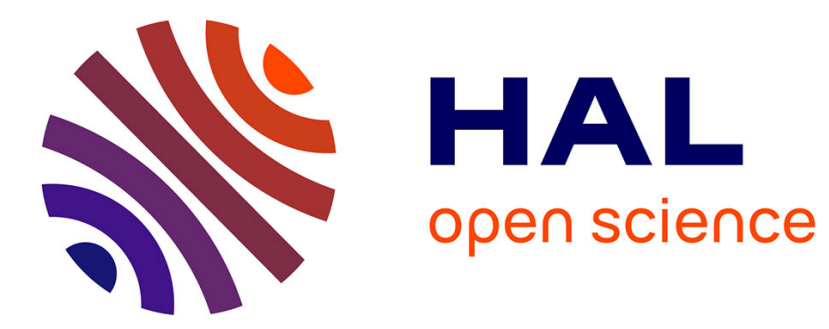

\title{
A multi-observers approach for a class of bidimensional non-uniformly observable systems
}

Ihab I. Haidar, Jean-Pierre Barbot, Alain Rapaport

\section{To cite this version:}

Ihab I. Haidar, Jean-Pierre Barbot, Alain Rapaport. A multi-observers approach for a class of bidimensional non-uniformly observable systems. 2020. hal-02903007

\section{HAL Id: hal-02903007 \\ https://hal.science/hal-02903007}

Preprint submitted on 20 Jul 2020

HAL is a multi-disciplinary open access archive for the deposit and dissemination of scientific research documents, whether they are published or not. The documents may come from teaching and research institutions in France or abroad, or from public or private research centers.
L'archive ouverte pluridisciplinaire HAL, est destinée au dépôt et à la diffusion de documents scientifiques de niveau recherche, publiés ou non, émanant des établissements d'enseignement et de recherche français ou étrangers, des laboratoires publics ou privés. 


\title{
A multi-observers approach for a class of bidimensional non-uniformly observable systems *
}

\author{
Ihab Haidar $^{1}$, Jean-Pierre Barbot ${ }^{1,2}$ And Alain Rapaport ${ }^{3}$ \\ ${ }^{1}$ Laboratoire Quartz EA 7393, ENSEA, Cergy-Pontoise, France. \\ 2 LS2N-CNRS UMR 6004, Nantes, France. \\ ${ }^{3}$ MISTEA, Univ Montpellier, INRAE, France.
}

July 19, 2020

\begin{abstract}
We consider the observation problem for a particular class of bidimensional systems with scalar output which requires the construction of an embedding in higher dimension. We propose a new approach that does not require any coordinates transformation. This approach is based on the design of parallel estimators in the same dimension than the original system. Each estimator uses the knowledge of the first two derivatives of the output, and the further derivatives are used to discriminate at any time among the different estimators. We give three examples showing the applicability of this approach with measurement noise. Biological systems used in batch bioprocess models are of particular motivation for this work.
\end{abstract}

Keywords Nonlinear systems, Observability, State observer, Singularity, Biological systems.

\section{Introduction}

The problem of observability and design of observers for finite dimensional dynamical systems has been attracted many attention in the literature of nonlinear control theory. The importance of this problem arises from many practical applications where the design of state estimators is needed [16]. One of the main and classical approach to obtain an observer in the original coordinates consists in transforming the original system into the so called observability form [8], determine an observer in this canonical form, and then expressing the estimation back in the original coordinates. Knowing that, in general, this change of coordinates is defined through an immersion in higher dimension space, and not simply a diffeomorphism, this approach has been widely investigated in the literature (see, e.g., $[1,3,4,5,6,12,13,17,19]$ ). The principal difficulty lies in the construction of an embedding and a Lipschitzian extension of the dynamics outside the set of its forward orbits. Another approach allowing the construction of local asymptotic observers without passing by the canonical form has been recently developed in [2].

In this paper, we propose another approach to deal with the problem of immersion in higher dimension space. We focus on the particular class of systems defined on a subset $\mathcal{S}$ of $\mathbb{R}^{2}$

$$
\begin{aligned}
& \dot{x}_{1}=f_{1}\left(x_{1}, x_{2}\right), \\
& \dot{x}_{2}=f_{2}\left(x_{1}, x_{2}\right),
\end{aligned}
$$

where $f_{1}$ is a rational function and $f_{2}$ is a sufficiently smooth function, along with the observation

$$
y(t)=x_{1}(t), \quad \forall t \geq 0 .
$$

\footnotetext{
*This work is supported by a public grant overseen by the Research and Valorization Service (SRV) of ENSEA.
} 
Instead of a single observer in higher dimension, we propose a set of observers in the original coordinates and a test function, based on higher derivatives of the observation, which can discriminate between the observers the one that will give the right estimate. More precisely, starting from equation (1), we build a set of estimators, each of them follows dynamically one and only one root of equation (1) and then use the further derivatives of the output together with equation (2) to select at any time the right estimator. This approach does not require any coordinates transformation, and by consequence no problem of transformation inversion of the observability map nor its Jacobian is posed. The technical task with this approach lies in the roots tracking method which, as described in the preliminary result of this work (see [10]), needs to be robust near singularities (multiple roots); robust in the sense that it always distinguishes between different neighboring roots. Indeed, knowing that each estimator should have a subset of (dynamical) roots as a global attractor and knowing that such attractors intersect at multiple roots, then, close to singularities, two distinct estimators may exchange roles and even fuse together. In addition, a relevant roots tracking method should take into account the initialization problem. In fact, an a priori knowledge of the different roots at initial time is needed. This can be possible if we deal with complex roots of equation (1). Here, we propose a robust roots tracking method based on a singular perturbation of equation (1) allowing the construction of its complex roots.

Knowing that the perfect knowledge of the output and its successive derivatives is hardly accessible in practice, the performance of this multi-observers approach in the case of measurement noise is shown through numerical simulations. Even in the case where the inverse of the observability map is available, which is not an easy task in general, we show by simulation the advantage of our approach.

For general class of systems of dimension $n>2$ with scalar measurement $y$, observability singularities may occurs as functions of $\left(\dot{y}, \ddot{y}, \cdots, y^{(n-1)}\right)$. Here, for sake of clearness, we restrict our study to bidimensional systems, so the singularity appears only as a function of $\dot{y}$. Nevertheless, several biological systems which are subject to the evoked singularity observability problem may encompassed by system (1)-(2) (see, e.g., [9, 17]). The paper is organized as follows. In Section 2 we describe the new approach. In section 3 we describe our roots tracking methods and give the main results. Three examples showing the applicability of the proposed method are given in Section 4.

\section{A multi-observers approach}

Let $\mathcal{D}$ be a relatively compact subset of $\mathcal{S}$ not containing the poles of $f_{1}$ and positively invariant by the dynamics (1)-(2). A sufficient condition for the construction of observers for system (1)-(2) on $\mathcal{D}$ is that the map $\left(x_{1}, x_{2}\right) \mapsto(y, \dot{y})$ is injective on $\mathcal{D}$ (see, e.g. [20]). When this condition loses to be satisfied somewhere on the validity domain, the usual constructions of observers in the system's dimension is not possible. Nevertheless, one can check the possibility of overcoming this singularity problem by considering further derivatives of the output. Indeed, if

$$
z=\Phi(x)=\left[h(x), L_{f} h(x), \cdots, L_{f}^{m-1} h(x)\right]^{T},
$$

where $f=\left(f_{1}, f_{2}\right)^{T}$ and $h(x)=x_{1}$, defines an injective immersion on $\mathcal{D}$, for some $m \geq 2$, the extension of system (1)-(2) to $\mathbb{R}^{m}$ is possible (see, e.g., [7]). In this case, a constructive method allowing the construction of an exponential observer for (1)-(2) on $\mathcal{D}$ in its original coordinates is proposed in [17].

Here, a different approach based on the design of several estimators in parallel is proposed. This approach is outlined in the sequel. Let $N$ and $D$ be the numerator and denominator of $f_{1}$, respectively. Observe that, since we suppose that $\mathcal{D}$ does not contain the poles of $f_{1}$ and that is positively invariant by the dynamics (1)$(2)$, a solution $s(t)$ of equation (1) must satisfy the following equation

$$
N(y(t), s(t))-\dot{y}(t) D(y(t), s(t))=0, \quad \forall t \geq 0 .
$$

The solution $s$ of (4) is, in general, not uniquely determined. But, since $N$ and $D$ are polynomial functions, then, for each fixed $z$ in $\mathbb{R}^{2}$, there exist at most $p$ solutions $s_{1}, \cdots, s_{p}$ such that

$$
F\left(z, s_{i}\right):=N\left(z_{1}, s_{i}\right)-z_{2} D\left(z_{1}, s_{i}\right)=0, \forall i=1, \cdots, p,
$$


where $p=\max \{\operatorname{deg}(N), \operatorname{deg}(D)\}$. Observe that the number of real solutions of (5) could depend on $z$. At any time $t \geq 0$, there exists at least one root $s_{i}$ such that $s(t)=s_{i}$. The multi-observers approach that we propose consists in computing dynamically $p$-parallel estimators $\hat{s}_{1}, \cdots, \hat{s}_{p}$ of these roots. If there are less than $p$ solutions of $F(z(t), s)=0$ at some time $t \geq 0$, then some estimators would be "temporarily" irrelevant. The way to determine these estimators will be addressed in the next section. The final task is to provide, at each $t \geq 0$, an estimation of $x_{2}(t)$ among $\hat{s}_{1}(t), \cdots, \hat{s}_{p}(t)$. For this purpose, using the further derivatives of $y$ and the injectivity of the map $\Phi$, we consider the test $\mathcal{T}(z, s)=0$, where the function $\mathcal{T}$ is defined as follows

$$
\mathcal{T}(z, s):=\left\|\left(z_{2}-\Phi_{2}\left(z_{1}, s\right), \cdots, z_{m}-\Phi_{m}\left(z_{1}, s\right)\right)\right\|_{M},
$$

where $\|\cdot\|_{M}$ denotes the norm associated to a real symmetric positive definite $(m-1) \times(m-1)$ matrix $M$, and from which we know that there exists only one solution among the (exact) solutions $s_{i}(t)$ at any time $t \geq 0$. In practice, we shall choose $\hat{s}(t)=\hat{s}_{i^{\star}(t)}(t)$ for which $\hat{s}_{i^{\star}(t)}(t)$ minimizes the function $\mathcal{T}\left(z(t), \hat{s}_{i}(t)\right)$ among the estimators $\left\{\hat{s}_{i}(t)\right\}_{i=1, \cdots, p}$, for $t \geq 0$. The choice of the norm plays a role when noisy output is considered; this will be discussed in Section 4.

\section{A roots tracking method}

Equation (5) is identically equal to zero over the solution of the dynamics (1)-(2). Then, starting from this equation, we define the following implicit dynamical system

$$
\left\{\begin{array}{l}
\frac{d}{d t} F(z(t), \hat{s}(t))=-K F(z(t), \hat{s}(t)), \quad \forall t \geq 0, \\
\hat{s}(0) \in \mathbb{R}
\end{array}\right.
$$

having the set

$$
S:=\{s \in \mathbb{R}: F(z(t), s)=0, t \geq 0\},
$$

as a global attractor, for $K>0$. Indeed, starting from an initial condition $\hat{s}_{0} \in \mathbb{R}$, we have

$$
F(z(t), \hat{s}(t))=F\left(z_{0}, \hat{s}_{0}\right) e^{-K t}, \quad \forall t \geq 0 .
$$

By consequence, a solution of (7) converges asymptotically to the set $S$. Moreover, the set $S$ is invariant by system (7). Observe that system (7) can be equivalently written as

$$
\left\{\begin{array}{l}
\partial_{s} F(z, \hat{s}) \dot{\hat{s}}=-\partial_{z} F(z, \hat{s}) \dot{z}-K F(z, \hat{s}), \\
\hat{s}(0) \in \mathbb{R} .
\end{array}\right.
$$

Three main problems prevent the construction of estimators of the roots of (4) from system (8):

a) The dynamics of $\hat{s}$ cannot be explicitly obtained from (8), especially when multiple solutions of (4) appear. In order to define the dynamics of $\hat{s}$ explicitly from (8), a preliminary solution has been proposed in [10] consists in saturating the norm of $\left(\partial_{s} F(z(t), \hat{s}(t))\right)^{-1}$ near the singularities.

b) Another problem is related to the initialization of the modified (saturated) dynamics related to system (8). In fact, seeing that the equation $F(z(0), \hat{s}(0))=0$ may have complex conjugate roots, an a priori information about the initialization of the corresponding solutions is needed.

c) An additional problem concerns the behavior of system (8) near the singularities. In fact, when multiple roots appear, i.e., when two neighboring solutions of (4) converge towards each other, the modified (saturated) dynamics related to system (8) cannot guaranty that the solutions, corresponding to $p$-different initial conditions, leave the cone $\left\{\left(s_{1}, \cdots, s_{p}\right) \in \mathbb{R}^{p}, s_{1}>s_{2}>\cdots>s_{p}\right\}$ invariant. By consequence, approaching a singularity two different estimators may merge into each other leading to loose a part of the solution. A preliminary solution based on an approximation dynamics coupling the relative vector fields corresponding to different initial conditions is proposed in [10]. 
In order to overcome the problems evoked previously, we consider the following perturbed implicit dynamics with complex variable

$$
\left\{\begin{array}{l}
\partial_{s} F_{\varepsilon}\left(z, \hat{s}_{\varepsilon}\right) \dot{\hat{s}}_{\varepsilon}=-\partial_{z} F_{\varepsilon}\left(z, \hat{s}_{\varepsilon}\right) \dot{z}(t)-K F_{\varepsilon}\left(z, \hat{s}_{\varepsilon}\right) \\
\hat{s}_{\varepsilon}(0) \in \mathbb{C}
\end{array}\right.
$$

where the function $F_{\varepsilon}: \mathbb{R}^{2} \times \mathbb{C} \rightarrow \mathbb{C}$ is defined, for $\varepsilon \geq 0$, by

$$
F_{\varepsilon}(z, s):=F(z, s)-\varepsilon \mathrm{i},
$$

and i denotes the imaginary unit number. Observe that for $\varepsilon$ arbitrarily small the roots of $F_{\varepsilon}(z, s)$ and $F(z, s)$ are arbitrarily close to each other (this will be clarified in the sequel). The dynamics (9) has the set

$$
S_{\varepsilon}=\left\{s \in \mathbb{C}: F_{\varepsilon}(z(t), s)=0, t \geq 0\right\},
$$

as a global attractor, for $K>0$. Indeed, from (9), we have

$$
F_{\varepsilon}\left(z(t), \hat{s}_{\varepsilon}(t)\right)=F_{\varepsilon}\left(z(0), \hat{s}_{\varepsilon}(0)\right) e^{-K t}, \quad \forall t \geq 0 .
$$

By consequence, a solution of (9) converges asymptotically to the set $S_{\varepsilon}$. Moreover, the set $S_{\varepsilon}$ is invariant by the dynamics (9). Indeed, starting from a (simple) root of $F_{\varepsilon}(z(0), s)$, an associated solution $s_{\varepsilon}$ will follow a root of $F_{\varepsilon}(z(t), s)$, for $t \geq 0$. The advantage of considering the perturbed dynamics (9) in $\mathbb{C}$, comparing to $(8)$ in $\mathbb{R}$, is summarized by the following three points:

a') The possible singularities of (9) are always complex. By consequence, seeing that $\partial_{s} F=\partial_{s} F_{\varepsilon}$, if we suppose that the solutions in $s$ of $\partial_{s} F(z, s)=0$ are always reals, the dynamics of $\hat{s}_{\varepsilon}$ can be explicitly and carefully defined from (9).

b') Knowing that the roots of $F_{\varepsilon}(z, s)$ are always complex, then, comparing to the point b), no problem of initialization of the dynamics (9) is present in this case.

c') The set $S_{\varepsilon}$ is composed of $p$-disjoints (simple) complex root subsets, each of them is associated to the set of simple complex roots of $F_{\varepsilon}(z(t), s)$, for $t \geq 0$. These $p$-disjoints subsets consist the $p$-different attractors of the dynamics (9). In addition, we can prove that the distance between two different neighboring attractors is lower bounded by a constant which is proportional to $\varepsilon$. By consequence, comparing to the point c), no problem of separation between two different neighboring solutions is present in this case.

Before proving the previously evoked points, let us first make the following assumption.

Assumption 1. For all $z \in \mathbb{R}^{2}$, the solutions of $\partial_{s} F(z, s)=0$ are always reals.

Observe that Assumption 1 can be relaxed by supposing that it holds only on the domain of validity of $z$, i.e., over $\Phi(\mathcal{D})$.

The following lemma discusses the well definition of dynamics (9) as an explicit dynamics of $\hat{s}_{\varepsilon}$.

Lemma 1. Suppose that Assumption 1 holds. Let $\varepsilon>0$ and $\hat{s}_{\varepsilon}$ be a solution of (9) starting from $\hat{s}_{0} \in \mathbb{C} \backslash \mathbb{R}$. We have that $\partial_{s} F_{\varepsilon}\left(z(t), \hat{s}_{\varepsilon}(t)\right) \neq 0$ for every $t \geq 0$.

Proof. Suppose by contradiction that $\partial_{s} F_{\varepsilon}\left(z(t), \hat{s}_{\varepsilon}(t)\right)=0$, for some $t \geq 0$. Then, under Assumption 1 , we have certainly that $\hat{s}_{\varepsilon}(t)$ is a real number. By consequence, we have

$$
\partial_{z} F\left(z(t), \hat{s}_{\varepsilon}(t)\right) \dot{z}(t)+K F\left(z(t), \hat{s}_{\varepsilon}(t)\right)=K \varepsilon \dot{\mathrm{i}},
$$

which is not possible because the left and right hand sides of equation (12) are real and complex, respectively. 
Observe that, under Assumption 1, the polynomial $F_{\varepsilon}(z, s)$ given by equation (10) does not have multiple complex roots (this is a property of holomorphic functions). By consequence, for each $z \in \mathbb{R}^{2}$, there exist $p$-distinct complex roots of the polynomial $F_{\varepsilon}(z, s)$. In order to simplify the analysis of the point c'), we consider the following root numbering: for each $\varepsilon \geq 0$ and each $t \geq 0$, let $s_{\varepsilon, 1}(t), \cdots, s_{\varepsilon, p}(t)$ be the complex solutions of $F_{\varepsilon}(z(t), s)=0$, lexicographically ordered from greater to less, i.e.,

$$
s_{\varepsilon, 1}(t) \succeq s_{\varepsilon, 2}(t) \succeq \cdots \succeq s_{\varepsilon, p}(t) .
$$

Knowing that, for each fixed $t \geq 0$, the roots of the polynomial $F_{\varepsilon}(z(t), s)$ depend continuously on its coefficients (see, e.g, [14]), we have that $s_{\varepsilon, i}(t) \rightarrow s_{0, i}(t)$ when $\varepsilon \rightarrow 0$, for $i=1, \cdots, p$. Let us denote by $S_{\varepsilon, i}$ the subset of $S_{\varepsilon}$ collecting the complex numbers $s_{\varepsilon, i}$, for $i=1, \cdots, p$. We have

$$
S_{\varepsilon}=\bigcup_{i=1}^{p} S_{\varepsilon, i} \quad \text { and } \quad S_{\varepsilon, i} \bigcap S_{\varepsilon, i+1}=\emptyset, \quad \forall i=1, \cdots, p,
$$

with $S_{\varepsilon, p+1}=\emptyset$. The fact that $S_{\varepsilon, i} \cap S_{\varepsilon, i+1}=\emptyset$, for all $1 \leq i \leq p$, derives straightforwardly from Assumption 1.

The following proposition clarifies the point c') concerning the separation between two neighboring solutions of (9). Let us first, for each $\varepsilon, T>0$, introduce the following set

$$
S_{\varepsilon}^{T}=\left\{s \in \mathbb{C}: F_{\varepsilon}(z(t), s)=0, t \in[0, T]\right\},
$$

which is a compact subset of $S_{\varepsilon}$.

Proposition 1. Suppose that Assumption 1 holds. Let $\bar{\varepsilon}, T>0$ and $L=L(F, \bar{\varepsilon}, T)$ be a Lipschitz constant associated to $F$ in the compact set $\bigcup_{0 \leq \varepsilon \leq \bar{\varepsilon}} S_{\varepsilon}^{T}$. We have

$$
\operatorname{dist}\left(S_{\varepsilon, i}^{T}, S_{\varepsilon, i+1}^{T}\right) \geq 2 \varepsilon / L, \quad \forall i=1, \cdots, p .
$$

Proof. Let $0 \leq \varepsilon \leq \bar{\varepsilon}$. Firstly, we prove that

$$
\operatorname{dist}\left(S_{\varepsilon, i}^{T}, S_{0, i}^{T}\right) \geq \varepsilon / L, \quad \forall i=1, \cdots, p .
$$

For this, let $s_{\varepsilon, i} \in S_{\varepsilon, i}^{T}$ and $s_{0, i} \in S_{0, i}^{T}$. Using the Lipschitzianity of $F$ over the set $\bigcup_{0 \leq \varepsilon \leq \bar{\varepsilon}} S_{\varepsilon}^{T}$, we have

$$
\left\|F\left(z, s_{\varepsilon, i}\right)-F\left(z, s_{0, i}\right)\right\| \leq L\left\|s_{\varepsilon, i}-s_{0, i}\right\| .
$$

Observe that

$$
\left\|F\left(z, s_{\varepsilon, i}\right)-F\left(z, s_{0, i}\right)\right\|=\left\|F_{\varepsilon}\left(z, s_{\varepsilon, i}\right)+\varepsilon \mathrm{i}-F\left(z, s_{0, i}\right)\right\|=\varepsilon .
$$

Thus, equation (15) leads to the following estimation

$$
\left\|s_{\varepsilon, i}-s_{0, i}\right\| \geq \varepsilon / L .
$$

By consequence, we have

$$
\operatorname{dist}\left(S_{\varepsilon, i}^{T}, S_{0, i}^{T}\right)=\min _{s_{\varepsilon, i} \in S_{\varepsilon, i}^{T}, s_{0, i} \in S_{0, i}^{T}}\left\|s_{\varepsilon, i}-s_{0, i}\right\| \geq \varepsilon / L
$$

Similarly, we have

$$
\operatorname{dist}\left(S_{\varepsilon, i+1}^{T}, S_{0, i+1}^{T}\right) \geq \varepsilon / L, \quad \forall i=1, \cdots, p .
$$

Observe that $S_{0, i}^{T}$ and $S_{0, i+1}^{T}$ are two conjugate solution sets, and that $S_{\varepsilon, i}^{T} \cap S_{0, j}^{T}=\emptyset$ for all $i, j=1, \cdots, p$, estimation (14) follows from inequalities (15)-(16). 
Remark 1. The set $S_{\varepsilon}^{T}$ is compact from the simple fact that it is closed $\left([0, T] \times S_{\varepsilon}^{T}=F_{\varepsilon}^{-1}(\{0\}) \cap([0, T] \times \mathbb{C})\right.$ with continuous function $F_{\varepsilon}$ ) and bounded (we use the fact that the roots of $F(z(t), s), t \in[0, T]$, are bounded together with the continuity dependency, with respect to $\varepsilon$, of the roots of $F_{\varepsilon}(z(t), s)$ ). The compacity of the set $\bigcup_{0 \leq \varepsilon \leq \bar{\varepsilon}} S_{\varepsilon}^{T}$ follows equally from the continuity dependency on $\varepsilon$ of the roots of $F_{\varepsilon}(z(t), s)$.

Now, we introduce the map $\mathcal{F}_{\varepsilon}: \mathbb{R}_{+} \times \mathbb{C} \rightarrow \mathbb{C}$, given, for each $(t, s) \in \mathbb{R}_{+} \times \mathbb{C}$, by

$$
\mathcal{F}_{\varepsilon}(t, s):=-\left(\partial_{s} F_{\varepsilon}(z, s)\right)^{-1}\left(\partial_{z} F_{\varepsilon}(z, s) \dot{z}(t)+K F_{\varepsilon}(z, s)\right),
$$

which, thanks to Lemma 1, is well defined. The dynamics (9) can be then equivalently written as

$$
\left\{\begin{array}{l}
\dot{\hat{s}}_{\varepsilon}(t)=\mathcal{F}_{\varepsilon}\left(t, \hat{s}_{\varepsilon}(t)\right), \quad \forall t \geq 0 \\
\hat{s}_{\varepsilon}(0) \in \mathbb{C}
\end{array}\right.
$$

The existence and uniqueness of solutions is clarified by the following lemma.

Lemma 2. Suppose that Assumption 1 holds. Then, for each $\varepsilon>0$, system (17) with initial condition $s_{0} \in S_{\varepsilon}$, has a unique solution over $\mathbb{R}_{+}$.

Proof. Let $\varepsilon>0$ and $s_{0} \in S_{\varepsilon}$. Knowing that the function $\mathcal{F}_{\varepsilon}$ is smooth, then there exists $t_{0}>0$ such that system (17) has a unique solution over $\left[0, t_{0}\right)$ starting from $s_{0}$. Knowing that the set $S_{\varepsilon}^{t_{0}}$, introduced by (13), is compact and that the solution lies entirely in $S_{\varepsilon}^{t_{0}}$, one can prove using a contradiction reasoning that $t_{0}=+\infty$ (see, e.g., [11]).

The following theorem shows the uniform convergence of the solutions of (17) to the roots of (5).

Theorem 1. Suppose that Assumption 1 holds. Then, for each $T, \delta>0$ there exists $\bar{\varepsilon}>0$ such that the solution of

$$
\begin{cases}\dot{\hat{s}}_{\varepsilon, i}(t)=\mathcal{F}_{\varepsilon}\left(t, \hat{s}_{\varepsilon, i}(t)\right), & t \geq 0, \\ \hat{s}_{\varepsilon, i}(0) \in S_{\varepsilon, i}, & i \in\{1, \cdots, p\},\end{cases}
$$

satisfies the following inequality

$$
\sup _{t \in[0, T]}\left|s_{\varepsilon, i}(t)-s_{0, i}(t)\right|<\delta, \quad \forall \varepsilon \in[0, \bar{\varepsilon}) .
$$

Proof. Let $T, \delta>0$. Knowing that the roots of a polynomial depends continuously on its coefficients (see, e.g, [14]), then for each $t \in[0, T]$, there exists an $\bar{\varepsilon}(\delta, z(t))>0$ such that the $p$-distinct roots of $F_{\varepsilon}\left(z(t), s_{\varepsilon}(t)\right)$ satisfy the following estimates

$$
\left|s_{\varepsilon, i}(t)-s_{0, i}(t)\right|<\delta, \quad \forall \varepsilon \in[0, \bar{\varepsilon}), i \in\{1, \cdots, p\} .
$$

Knowing that, for $t \in[0, T], z(t)$ lies in a compact subset of $\mathbb{R}^{2}$, then, by compacity reasoning, there exists $\bar{\varepsilon}=\bar{\varepsilon}(\delta, T)$ such that inequality (19) holds. Hence, the proof of the theorem.

We have the following theorem.

Theorem 2. Consider system (1)-(2). Let $\mathcal{D}$ be a positively invariant compact subset of $\mathbb{R}^{2}$ not containing the poles of $f_{1}$. Suppose that the map $\Phi$, given by (3), is injective over $\mathcal{D}$, for some $m \geq 2$. Suppose that Assumption 1 holds. Then, system (1)-(2) is observable over $\mathcal{D}$.

Proof. The proof is a straightforward consequence of Theorem 1. We have to underline that even if the solutions of the proposed estimators (18) crosses the poles of $f_{1}$, this will be for a null set of time.

\section{Examples}

In this section we give three examples showing the applicability of our method to deal with non-uniformly observable bidimensional systems. 

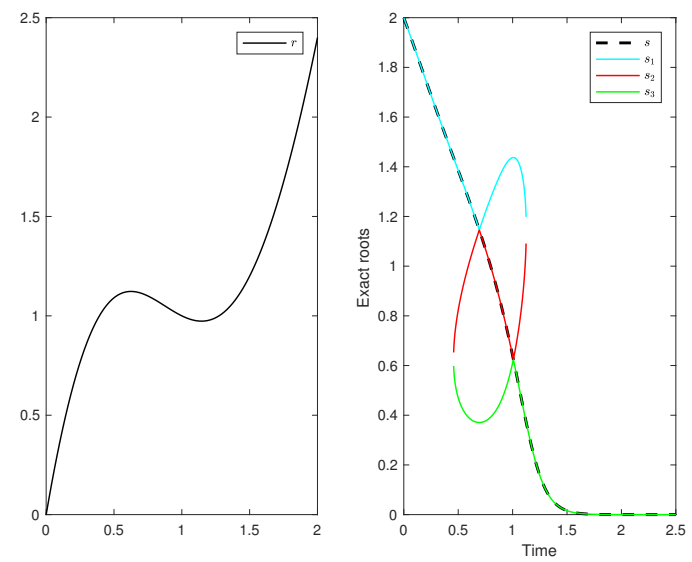

Figure 1: Left: the function $r$ given by (22). Right: the exact roots of (5).

\subsection{Example 1}

Let us consider the following dynamics in $\mathbb{R}_{+}^{2}$

$$
\begin{aligned}
& \dot{x}_{1}=x_{1} r\left(x_{2}\right), \\
& \dot{x}_{2}=-x_{1} r\left(x_{2}\right),
\end{aligned}
$$

where $r: \mathbb{R}_{+} \rightarrow \mathbb{R}_{+}$is given by

$$
r(s)=\frac{6 s\left(s^{2}-2.5 s+2\right)}{s^{2}-s+3},
$$

along with the observation

$$
y(t)=x_{1}(t), \quad \forall t \geq 0 .
$$

It is easy to check that the positive orthant is invariant by (20)-(21) and that the poles of $r$ does not belong there. In addition, we have

$$
x_{1}(t)+x_{2}(t)=x_{1}(0)+x_{2}(0), \quad \forall t \geq 0,
$$

which implies that, starting from the positive orthant, the trajectories of system (20)-(21) stay bounded when $t$ tends to $+\infty$. The construction of $x_{2}$ from (20) requires to solve the equation $r(s)=\dot{y}(t) / y(t)$ over $\mathbb{R}_{+}$, whose real solution numbers varies in times, as one can observe from Figure 1-right. In order to construct the solutions of the latest equation, we consider its corresponding perturbed dynamics (9) with

$$
F(z, s)=6 z_{1} s\left(s^{2}-2.5 s+2\right)-\left(s^{2}-s+3\right) z_{2} .
$$

The roots tracking method proposed in Section 3 consists in simulating the corresponding perturbed dynamics starting from the roots of $F_{\varepsilon}(z(0), s)$, for some $\varepsilon>0$. The three different solutions $\hat{s}_{\varepsilon, 1}(t), \hat{s}_{\varepsilon, 2}(t)$ and $\hat{s}_{\varepsilon, 3}(t)$ (which are always complex) will follow the roots of $F_{\varepsilon}(z(t), s)$, for $t \geq 0$. In order to construct an estimation of $s_{\varepsilon}(t)$, we have to determine among the 3 constructed solutions which one is the right one, at any $t \geq 0$. For this, we choose the solution $\hat{s}_{\varepsilon}(t)=\hat{s}_{\varepsilon, i^{\star}(t)}(t)$ for which $\Re\left(\hat{s}_{\varepsilon, i^{\star}(t)}(t)\right)$ minimizes the function $\mathcal{T}\left(z(t), \Re\left(\hat{s}_{\varepsilon, i}(t)\right)\right)$ among the set $\left\{\hat{s}_{\varepsilon, i}(t)\right\}_{i=1,2,3}$, where $\Re\left(\hat{s}_{\varepsilon, i}(t)\right)$ denotes the real part of $\hat{s}_{\varepsilon, i}(t)$. The choice of the norm in (6) plays an important role when dealing with numerical differentiators, especially when measurement noise is considered. In fact, when some a priori knowledge on the nature of the noise is known, one could determine numerically a covariance matrix of the estimation error (cf., e.g., [18]) of the time derivatives of the observation, whose inverse can be chosen for the norm in (6). Typically, one expects to 


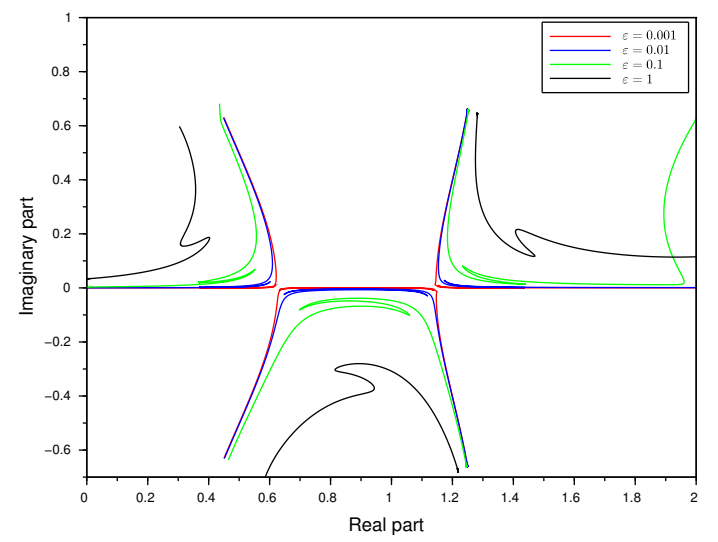

Figure 2: Illustration of Proposition 1. We represent the three solution sets relative to (23). We plot $s_{r}$ with respect to $s_{c}$ for $\varepsilon \in\{0.001,0.01,0.1,1\}$.

have lower weight for high order derivatives whose estimation is more prone to be affected by measurement noise. In the absence of noise, the identity matrix can be simply used to define the norm.

One can check, using the expression of the function $r$, the injectivity of the map $\Phi$, given by (3), with $m=3$. Observe also that Assumption 1 holds in this case. Indeed, we have

$$
\partial_{s} F(z, s)=18 z_{1} s^{2}-2\left(15 z_{1}+z_{2}\right) s+12 z_{1}+z_{2},
$$

for which one can easily verify that, for each $z_{1}, z_{2} \geq 0$ the solutions of $\partial_{s} F(z, s)=0$ are always reals. We simulate system (18) starting from the initial conditions of $F_{\varepsilon}(z(0), s)=0$, for $i=1,2,3$ and for different values of $\varepsilon$. The initial conditions of the original system $(20)-(21)$ are fixed at $(0.5,2)$. The parameter $K$, relative to the estimators (18) has been fixed to $K=150$. An explicit Euler scheme with a discretization step equal to $h=10^{-3}$ has been chosen for the simulation.

Assuming the perfect knowledge of $z$, that is the perfect knowledge of the derivative of the output $y$, we firstly illustrate the result given by Proposition (1) concerning the separation between neighboring solutions around the singularities. We show by Figure 2 the solution sets given by (11) corresponding to (23) for different value of the perturbation parameter $\varepsilon$. We clearly observe the role of $\varepsilon$ on separating neighboring solutions near the singularities. More precisely, as proved by Proposition 1, the distance between the solution sets is somehow proportional to the magnitude of $\varepsilon$. By Figure 3 we show the different real and complex parts of the three roots together with the constructed solution, where the perturbation parameter $\varepsilon$ is taken equal to 0.01 (left) and 0.1 (right).

Here, we have assumed the perfect knowledge of the vector $z(t)$ at any time $t \geq 0$, which is hardly accessible in practice. For this, we simulate our estimators where the derivatives of the output are given by a high-gain differentiator. In addition, we consider that the output measurements are randomly disturbed by a white noise proportional up to $5 \%$ of $x_{1}$. In order to check the robustness of the proposed approach to estimate the exact roots of (23), we simulate system (18) in the same condition as before. The output as well as its numerical derivatives are filtered offline using moving average filters. We also consider a short delay before computing the estimators $\hat{s}_{\varepsilon, i}$, the time for the differentiator to converge. As it is already mentioned, the perturbation parameter $\varepsilon$ plays a crucial role in separating neighboring solutions. This is particularly interesting in the case of measurement noise, where a sufficiently large $\varepsilon$ allows the separation of the disturbed estimators $\hat{s}_{\varepsilon, i}$ around the singularities. Of course, a large value for $\varepsilon$ acts against the precision of the estimator (18), and this should be fixed depending on the amplitude of the noise. Concerning the test function, knowing that $z_{3}$ is more affected by the noise measurement than $z_{2}$, we have simply chosen 

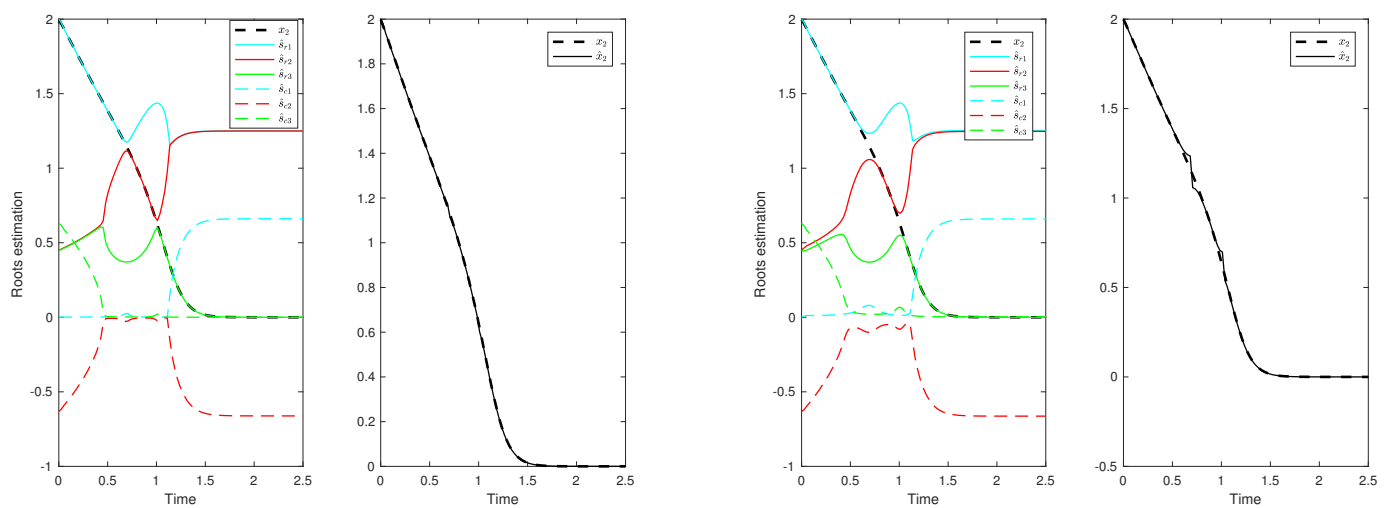

Figure 3: Illustration of the estimated roots of (23) with perfect knowledge of $z$ together with the exact and the constructed solution with $\varepsilon=0.01$ (left) and $\varepsilon=0.1$ (right).
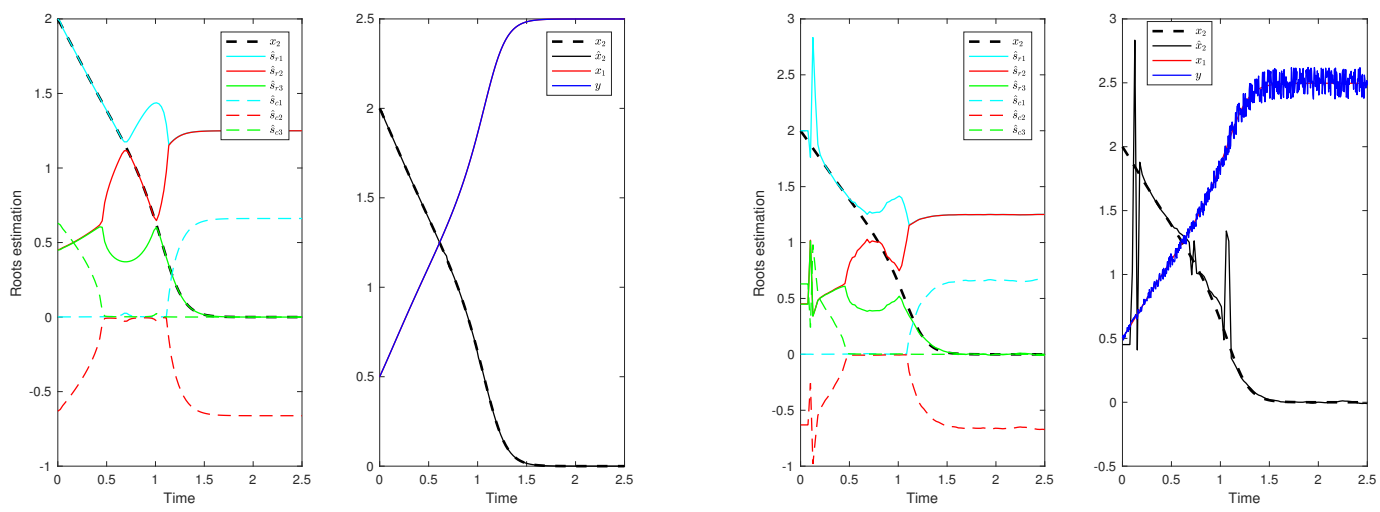

Figure 4: Illustration of the estimated roots of (23) together with the exact and the constructed solution (with $\varepsilon=0.01$ ), in the case of estimated output's derivatives without noise (left) and with white noise proportional up to $5 \%$ of $x_{1}$ (right).

$M=\operatorname{diag}(1,0.1)$ to define the norm in (6). By Figure 4 we show the different real and complex parts of the estimated roots of (23) together with the estimation of $x_{2}$ where $\varepsilon$ is fixed at 0.01 .

\subsection{Example 2}

An important class of bioprocesses, which is mainly used in food and pharmaceutical industry, is the batch bioreactor [15]. This bioprocesses is characterized by the fact that after the initial charge of the substrate in the bioreactor, there is no inflow or outflow of the medium. The typical model characterizing the substrate biodegradation in a batch culture is given by the same system (20)-(21), where in this case the variables $x_{1}$ and $x_{2}$ represent the biomass and substrate concentration, respectively. The function $r$, representing the microbial growth rate function, is given in this case by

$$
r\left(x_{2}\right)=\frac{k_{1} s}{k_{2}+s+k_{3} s^{2}}
$$



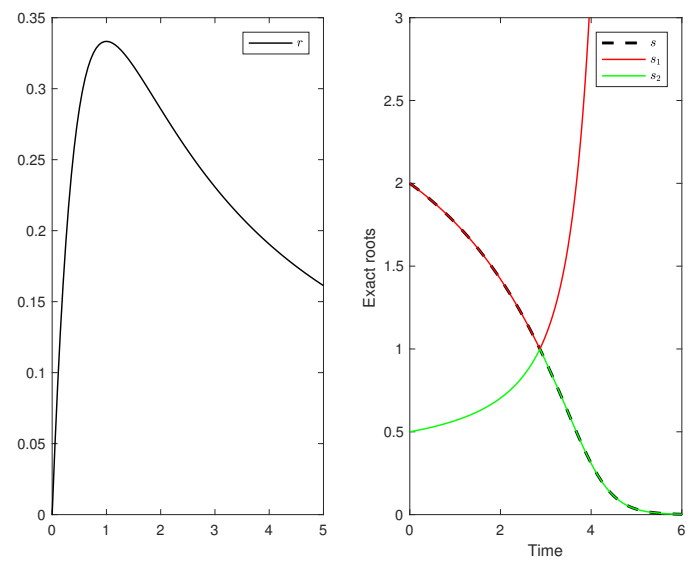

Figure 5: Left: the function $r$ given by (24). Right: the exact roots of (5).

where $k_{1}, k_{2}$ and $k_{3}$ are positive constants. Without loss of generality, we assume here that the yield coefficient of the transformation of the substrate into biomass is equal to 1 . Several works in the literature aim to reconstruct the substrate concentration when only the biomass concentration is measured (see, e. g., $[7,17])$. In the case when the function $r$ is non-monotonic, usually related to some inhibition effect of the reaction, this observability problem becomes particularly difficult. In fact, in this case, a singularity observability problem appears in the state space, more precisely over the set $\left\{\left(x_{1}, x_{2}\right) \in \mathbb{R}_{+}^{2}: x_{2}=\sqrt{k_{2} k_{3}}\right\}$. However, the system is observable but with an injectivity index equal to 3 . This problem is treated in (see, e.g., [17]) with the usual immersion approach described in the introduction. In order to show the applicability of our method in this case, we repeat the same analysis as in Example 1 with the function $r$ given by (24), with $k_{1}=k_{2}=k_{3}=1$. By Figure 6 we show the different real and complex parts of the estimated roots together with the estimation of the solution of $x_{2}$ where $\varepsilon$ is fixed at 0.01 , in the case of estimated $z$ without noise (left) and with noise measurement proportional up to $5 \%$ of $x_{1}$ (right). The identity matrix $M=I_{2}$ is simply chosen to define the test function in this case. It is worth noting that the estimators become quite sensitive to noise near the equilibrium point. This is explained by the fact that the exact derivatives become practically null close to the equilibrium point (this difficulty was already present with the approach proposed in $[17])$.

We end this section by underlying the advantage of the proposed approach to deal with singularities. For this, we compare the constructed solution together with the one obtained directly by inverting the observability map $\Phi$. A straightforward computation gives

$$
\Phi^{-1}(z)=\left(z_{1}, \frac{2 h_{1}^{3}(z)+h_{1}(z) h_{2}(z)}{\left(1-h_{1}(z)\right)\left(h_{1}^{2}(z)+h_{2}(z)\right)}\right)^{T},
$$

where

$$
h_{1}(z)=\frac{z_{2}}{z_{1}}, \quad h_{2}(z)=\frac{z_{2}^{2}-z_{1} z_{3}}{z_{2} z_{1}^{2}} .
$$

By Figure 7 we compare the solution constructed by our method, with $\varepsilon=0.01$, together with that obtained by $\Phi^{-1}$, in the case of estimated $z$, with white noise proportional up to $0.001 \%$ of $x_{1}$ and without any filtering strategy. We see clearly the performance of our approach in this case. 

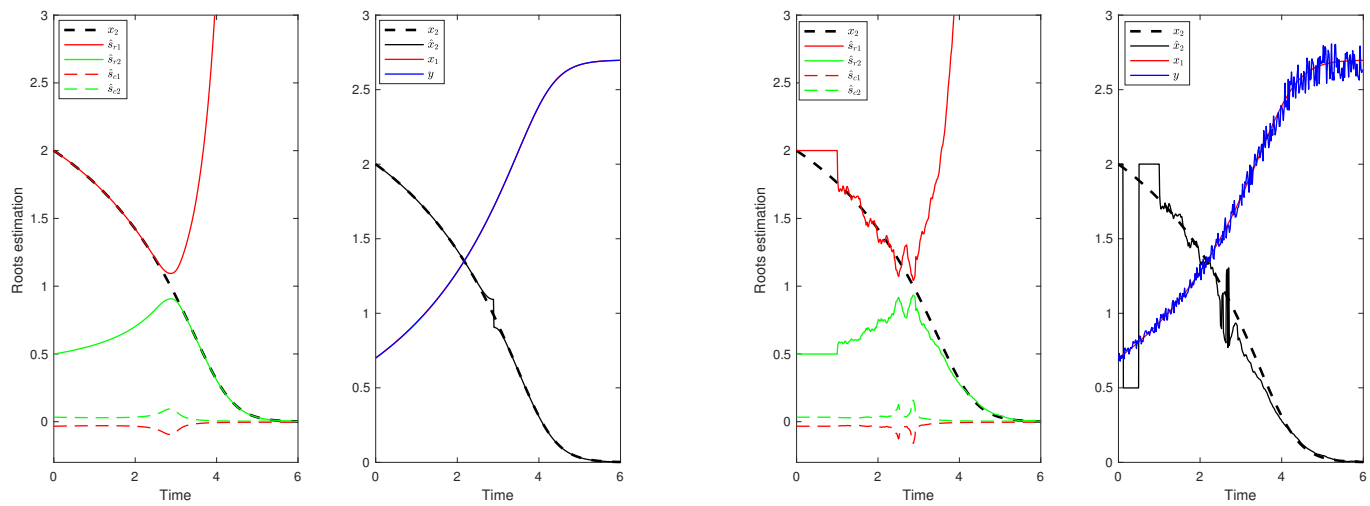

Figure 6: Illustration of the estimated roots corresponding to Example 2 together with the exact and the constructed solution (with $\varepsilon=0.01$ ). Case of estimated $z$ without noise (left) and with noise measurement proportional up to $5 \%$ of $x_{1}$ (right).

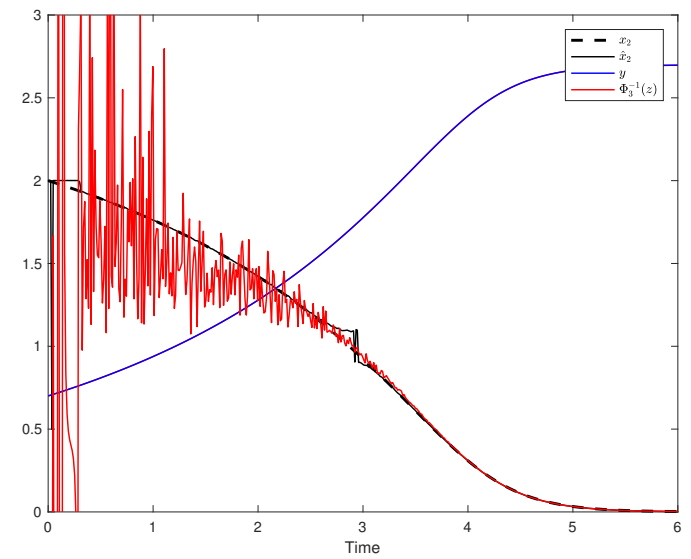

Figure 7: The exact solution together with the constructed one (with $\varepsilon=0.01$ ) and that obtained with $\Phi^{-1}$. Case of estimated $z$ with noise measurement proportional up to $0.001 \%$ of $x_{1}$ and without any filtering strategy.

\subsection{Example 3}

Let us consider the following dynamics in $\mathbb{R}^{2}$

$$
\begin{aligned}
& \dot{x}_{1}=a x_{2}-\left(x_{1}^{2}+x_{2}^{2}-a^{2}\right), \\
& \dot{x}_{2}=-a x_{1},
\end{aligned}
$$

along with the on-line observation

$$
y(t)=x_{1}(t),
$$

where $a$ is a positive real number. It is easy to see that the ball $B(0, a)$ is invariant by the dynamics $(25)-$ (26). In the case of this example, the construction of $x_{2}$ from (25) presents a singularity at $x_{2}=a / 2$. The 

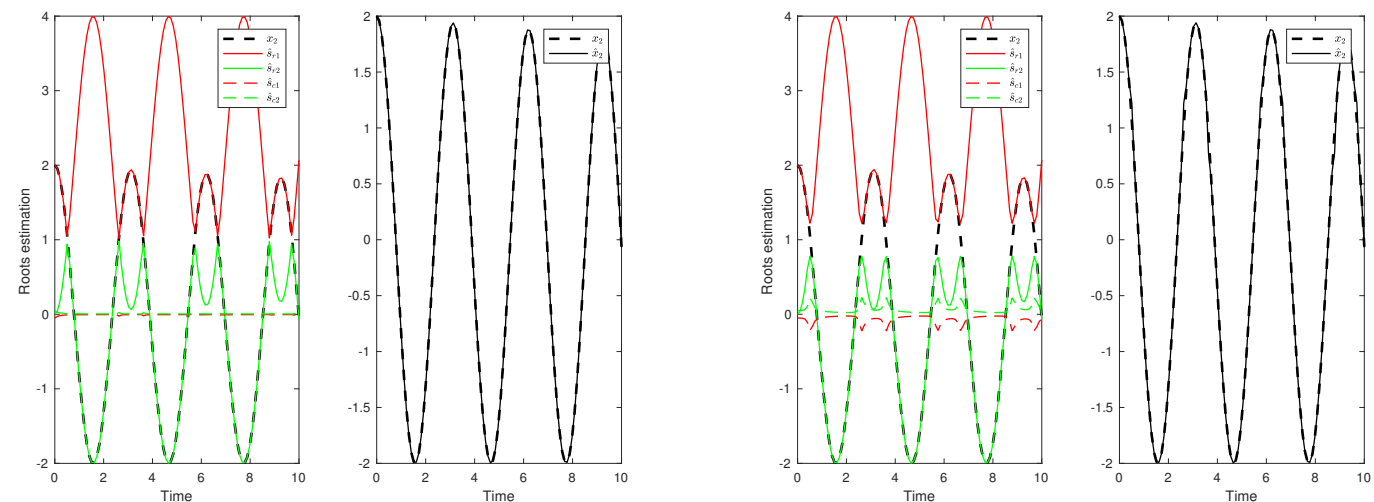

Figure 8: Illustration of the estimated roots corresponding to Example 3 with perfect knowledge of $z$ together with the exact and the constructed solution with $\varepsilon=0.001$ (left) and $\varepsilon=0.1$ (right).
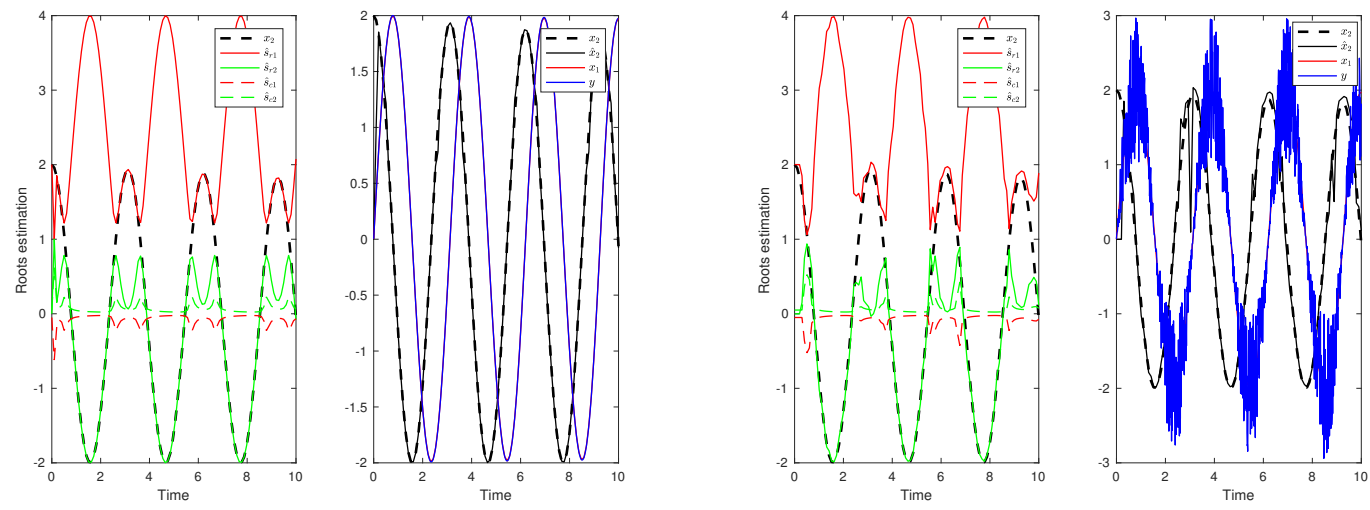

Figure 9: Illustration of the estimated roots corresponding to Example 3 together with the exact and the constructed solution (with $\varepsilon=0.1$ ). Case of estimated $z$ without noise (left) and with noise measurement proportional up to $50 \%$ of $x_{1}$ (right).

problem here is that this singularity problem may recurs periodically over the time. For the construction of $x_{2}$, we repeat the same analysis as in Example 1. For this, we define firstly the function

$$
F(z, s)=-s^{2}+a s-z_{1}^{2}-z_{2}+1 .
$$

We can easily verify that Assumption 1 is satisfied. In order to show the applicability of our approach in this case of periodic singularity, we simulate system (18) starting from the initial conditions of $F_{\varepsilon}\left(z(0), s_{\varepsilon, i 0}\right)=0$, for $i=1,2$ and different values of $\varepsilon$. The value of $a$ is fixed to $a=2$ and the initial condition of the original system (25)-(26) is fixed at $(0,2)$. Assuming the perfect knowledge of $z$, by Figure 8 we show the different real and complex parts of the estimated roots together with the constructed solution, where the perturbation parameter $\varepsilon$ is taken equal to 0.001 (left) and 0.1 (right). Now, without assuming the perfect knowledge of $z$, and using the same procedure as in Example 1 to give an estimation of it, we simulate system (18), where $\varepsilon$ is fixed at 0.1. By Figure 9 we show the different real and complex parts of the estimated roots together with the estimation of $x_{2}$ in two cases: without noise measurement (left) and with noise measurement proportional up to $50 \%$ of $x_{1}$ (right). The identity matrix $M=I_{2}$ is simply chosen to define the test function. 


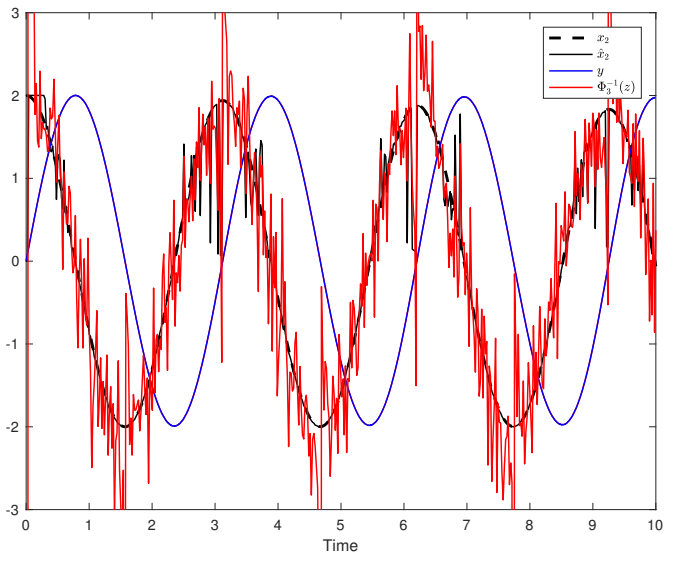

Figure 10: The exact solution together with the constructed one (with $\varepsilon=0.1$ ) and that obtained with $\Phi^{-1}$. Case of estimated $z$ with noise measurement proportional up to $0.2 \%$ of $x_{1}$ and without any filtering strategy.

As in Example 2, we compare the constructed solution together with the one obtained directly by inverting the observability map $\Phi$. A straightforward computation gives

$$
\Phi^{-1}(z)=\left(z_{1}, \frac{a^{2}+2 z_{2}+z_{3} / z_{1}}{2 a}\right)^{T} .
$$

By Figure 10 we show the solution constructed by our method, with $\varepsilon=0.1$, together with the one obtained by $\Phi^{-1}$, in the case of estimated $z$, with white noise proportional up to $0.2 \%$ of $x_{1}$. This comparison is done without using any filtering strategy. The performance of our approach is once again shown by this comparison.

\section{Conclusion}

In this work we propose a new approach to deal with a family of observability problems which, in general, requires the construction of embedding in higher dimensional space. This approach does not require any change of coordinates, and consequently there is no need to inverse the observability map nor its Jacobian. This relies on the following idea: instead of single observer in higher dimension, a set of estimators can be constructed in the original space together with a test function, based on higher derivative of the observation, to discriminate between the different estimators at any time; the right one is the one minimizing the test function. The choice of the norm for the test function gives flexibility to suit to the nature of the noise in the case of noisy measurements. Although this study is reduced to a particular class of bidimensional systems, non-uniform observability encountered in biological systems can be overcome by our approach. Future works will attempt to extend this work to more general class of higher dimensional autonomous systems.

\section{References}

[1] A. Astolfi, R. Ortega and A. Venkatraman, A globally exponentially convergent immersion and invariance speed observer for mechanical systems with non-holonomic constraints, Automatica, 46(1), 182-189, 2010. 
[2] D. Astolfi and C. Possieri, Design of local observers for autonomous nonlinear systems not in observability canonical form, Automatica, 103, 443-449, 2019.

[3] J. Back and J. Seo, An algorithm for system immersion into nonlinear observer form: SISO case, Automatica, 42(2), 321-328, 2006.

[4] P. Bernard, V. Andrieu and L. Praly, Expressing an Observer in Preferred Coordinates by Transforming an Injective Immersion into a Surjective Diffeomorphism, SIAM J. Control Optim., 56(3), 2327-2352, 2018.

[5] P. Bernard, L. Praly, V. Andrieu and H. Hammouri, On the triangular canonical form for uniformly observable controlled systems, Automatica, 85, 293-300, 2017.

[6] G. Besancon and A. Ticlea, An Immersion-Based Observer Design for Rank-Observable Nonlinear Systems, IEEE Transactions on Automatic Control, 52, 83-88, 2007.

[7] J.P. Gauthier, H. Hammouri and I. Kupka, Observers for nonlinear systems, IEEE Conference on Decision and Control (CDC), Brighton, England, 1991.

[8] J.P. Gauthier and I. Kupka, Deterministic Observation Theory and Applications. Cambridge University Press, 2001.

[9] I. Haidar, J. P. Barbot, A. Rapaport and M. Ghanes, Observability singularity of batch bioreactors: A solution based on high order sliding mode differentiator approach, 11th Asian Control Conference (ASCC), 336-338, 2017.

[10] I. Haidar, J. P. Barbot and A. Rapaport, A multi observers approach when observability index is higher than the state dimension-a case study-, 58th IEEE Conference on Decision and Control (CDC), 2019.

[11] H. K. Khalil, Nonlinear Systems, Prentice-Hall, 2002.

[12] J. Levine and R. Marino, Nonlinear system immersion, observers and finite-dimensional filters, Systems \& Control Letters, 7(2), 133-142, 1986.

[13] K.A.A. Langueh, O. Datcu, J.P. Barbot, G. Zheng, K. Busawon, Observabilty singularities and observer design: dual immersion approach, IFAC-PapersOnLine, 49(18), 511-516, 2016.

[14] R. Naulin and C. Pabst The roots of a polynomial depend continuously on its coefficients. Revista Colombiana de Matematicas, 28(1), 35-37, 1994.

[15] J.Pirt, Principles of microbe and cell cultivation. John Wiley and Sons, 1976.

[16] A. Rapaport and D. Dochain, A robust asymptotic observer for systems that converge to unobservable states. A batch reactor case study, IEEE Transactions on Automatic Control, 65(6), 2693-2699, 2020.

[17] A. Rapaport and M. Maloum, Design of exponential observers for nonlinear systems by embedding, International Journal of Robust and Nonlinear Control, 14, 273-288, 2004.

[18] G. Seber and C. Wild, Nonlinear regression, Wiley, 2003.

[19] A. Ticlea and G. Besancon, Immersion-Based Observer Design, In: Nonlinear Observers and Applications, Lecture Notes in Control and Information Sciences 363, (Ed. G. Besancon), Springer, 2007.

[20] X. Xia and M. Zeitz, On nonlinear continuous observers, International Journal of Control, 66(6), 943954, 1997. 